C. Rotthaus

Nagoya Math. J.

Vol. 76 (1979), 173-180

\title{
KOMPLETTIERUNG SEMILOKALER QUASIAUSGEZEICHNETER RINGE
}

\author{
CHRISTEL ROTTHAUS
}

In [4] EGA IV (7.4.8) hat Grothendieck die folgende Frage gestellt: " $A$ sei ein noetherscher Ring, $I \subseteq A$ ein Ideal, so daß $A$ separiert und komplett in der $I$-adischen Topologie ist. $A / I$ sei ein $P$-Ring. Ist dann $A$ ebenfalls ein $\boldsymbol{P}$-Ring?" In dieser Arbeit beschäftigen wir uns mit dem Fall, daß $A$ ein semilokaler noetherscher Ring ist und $\boldsymbol{P}$ die Eigenschaft "die formellen Fasern von $A$ sind geometrisch regulär" bezeichnet. Wir wollen zeigen: " $A$ sei ein semilokaler noetherscher $I$-adisch kompletter Ring, wobei $I$ ein im Jacobsonradikal von $A$ enthaltenes Ideal ist. Sind die formellen Fasern von $A / I$ geometrisch regulär, so sind auch die formellen Fasern von $A$ geometrisch regulär."

Im folgenden nennen wir einen semilokalen noetherschen Ring $A$ quasiausgezeichnet, wenn seine formellen Fasern geometrisch regulär sind. Unter dem Radikal $\operatorname{rad}(A)$ eines Ringes $A$ verstehen wir immer das Jacobsonradikal von $A$ und mit $\hat{A}$ werde die Komplettierung von $A$ nach der vom Jacobsonradikal auf $A$ induzierten Topologie (auch einfach Komplettierung von $A$ genannt) bezeichnet. Bei den übrigen Bezeichnungen sei auf EGA [3] und [4] bzw. das Buch von H. Matsumura [5] verwiesen.

Herrn Markus Brodmann danke ich für zahlreiche nützliche Gespräche über diese Arbeit.

\section{§1. Vorbereitungen}

Wir geben eine Zusammenstellung der zum Beweis des Hauptergebnisses benötigten Sätze:

Theorem 1 (Marot [7]). A sei ein semilokaler noetherscher Ring; $I \subseteq \operatorname{rad}(A)$ ein im Jacobsonradikal von A enthaltenes Ideal. A sei komplett in der I-adischen Topologie. Sind die formellen Fasern von A/I geometrisch reduziert, so sind die formellen Fasern von $A$ ebenfalls geometrisch

Received December 4, 1978. 
reduziert.

Theorem 2 (André [1]). $A$ und $B$ seien lokale noethersche Ringe, $\varphi: A \rightarrow B$ sei ein lokaler, in der Topologie der maximalen Ideale formell glatter Homomorphismus. Ist A quasiausgezeichnet, so lokalisiert die formelle Glattheit von $\varphi$.

Bemerkung. Die Aussage von Theorem 2 bedeutet: Für alle $\mathfrak{\beta} \in \operatorname{Spec}(B)$ ist der von $\varphi$ induzierte Morphismus $\varphi_{\mathfrak{\beta}}: A_{\mathfrak{p}} \rightarrow B_{\mathfrak{\beta}}$ (wobei $\mathfrak{p}=\varphi^{-1}(\mathfrak{P})$ ) ebenfalls formell glatt in der Topologie der maximalen Ideale. Insbesondere folgt unter den Bedingungen von Theorem 2, daß der Morphismus $\varphi: A$ $\rightarrow B$ regulär ist, d.h. $\varphi$ ist flach mit geometrisch regulären Fasern.

Ferner benötigen wir die folgende Charakterisierung quasiausgezeichneter semilokaler Ringe:

Lemma 1 ([5] (33.E)). A sei ein semilokaler noetherscher Ring. Dann sind folgende Aussagen äquivalent:

(a) A ist quasiausgezeichnet.

(b) Für alle nullteilerfreien endlichen A-Algebren $B$ ist für alle $\mathfrak{Q} \in \operatorname{Spec}(\hat{B})$ mit $\mathfrak{Q} \cap B=(0)$ der lokale Ring $\hat{B}_{\mathfrak{Q}}$ regulär.

Bemerkung.

1) Aus (b) in Lemma 1 folgt insbesondere, daß $A$ die Eigenschaft $J-2$ erfüllt (vgl. [5] (32.B)).

2) (33.E) ist nur für lokale Ringe formuliert. Der semilokale Fall ergibt sich jedoch als unmittelbare Folgerung.

Wir stellen nun einige beweistechnisch wichtige Hilfssätze zusammen:

Im folgenden sei $A$ immer ein noetherscher semilokaler Ring; $I \subseteq \operatorname{rad}(A)$ sei ein im Jacobsonradikal von $A$ enthaltenes Ideal.

LEMma 2. A sei I-adisch komplett. $\mathfrak{a} \subseteq \hat{A}$ sei ein vom Nullideal verschiedenes Ideal in $\hat{A}$. Für alle $n \in N$ mit $n \geqq n_{0}$ gelte: $\left(A \cap\left(\mathfrak{a}+I^{n} \hat{A}\right)\right) \hat{A}$ $=a+I^{n} \hat{A}$. Dann ist $a \cap A \neq(0)$.

Beweis. Wir setzen $\mathfrak{a}_{n}=\left(\mathfrak{a}+I^{n} \hat{A}\right) \cap A$. Nach Voraussetzung gilt für alle $n \geqq n_{0}: \mathfrak{a}_{n+1}+I^{n}=a_{n}$. Wegen $a \neq(0)$ ist $\mathfrak{a} \not \subset(\operatorname{rad}(\hat{A}))^{r}$ für ein $r \in N$ mit $r \geqq n_{0}$. Wähle $f_{r} \in \mathfrak{a}_{r} \backslash(\operatorname{rad}(A))^{r}$. Dann gibt es ein $f_{r+1} \in \mathfrak{a}_{r+1}$ mit $f_{r}-f_{r+1} \in I^{r}$, da $a_{r+1}+I^{r}=\mathfrak{a}_{r}$. Wir können also eine Folge $f_{n} \in \mathfrak{a}_{n}, n \geqq r$, finden mit $f_{n+1}-f_{n} \in I^{n}$ und $f_{n} \notin(\operatorname{rad}(A))^{r}$ für alle $n \geqq r$. Da $A$-adisch 
komplett ist, existiert $\lim _{n \rightarrow \infty} f_{n}=f \in A$ mit $f \neq 0$. Wegen $\bigcap_{n \geqq 0} \mathfrak{a}_{n} \subseteq \mathfrak{a} \cap A$ $f \in \mathfrak{a} \cap A \neq(0)$.

LEMMA 3. A sei I-adisch komplett. $a \subseteq \hat{A}$ sei ein Ideal mit $\sqrt{\mathfrak{a}+I \hat{A}}$ $=\bigcap_{j=1}^{r} \mathfrak{M}_{j}$, wobei die $\mathfrak{M}_{j}$ maximale Ideale in $\hat{A}$ sind. Dann folgt: $\mathfrak{a} \cap A \neq(0)$.

Beweis. Die Primärkomponenten von $a+I^{n} \hat{A}$ sind für alle $n \in N$ $\mathfrak{M}_{j}$-primär. Dann ist mit $\mathfrak{a}_{n}=\left(\mathfrak{a}+I^{n} \hat{A}\right) \cap A: \mathfrak{a}_{n} \hat{A}=\mathfrak{a}+I^{n} \hat{A}$, und die Behauptung folgt mit Lemma 2.

Ist $\varphi: A \rightarrow B$ ein lokaler Morphismus lokaler Ringe, so sagen wir im folgenden " $\varphi$ ist formell glatt", falls $\varphi$ in der Topologie der maximalen Ideale formell glatt ist.

Definition. $\quad \Gamma_{I}=\{(\mathfrak{p}, \mathfrak{P}) \mid \mathfrak{p} \in \operatorname{Spec}(A) ; \mathfrak{P} \in \operatorname{Spec}(\hat{A})$ mit $\mathfrak{P} \cap A=\mathfrak{p}$; $\mathfrak{p} \supseteq I$ und $\mathfrak{P}$ nicht maximal in $\operatorname{Spec} \hat{A}\} \subseteq \Gamma_{0}=\{(\mathfrak{p}, \mathfrak{P}) \mid \mathfrak{p} \in \operatorname{Spec}(A), \mathfrak{P} \in \operatorname{Spec}(\hat{A})$ $\operatorname{mit} \mathfrak{R} \cap A=\mathfrak{p}\} \subseteq \operatorname{Spec}(A) \times \operatorname{Spec}(\hat{A})$.

Lemma 4. A/I sei quasiausgezeichnet. Dann ist für alle $(\mathfrak{p}, \mathfrak{\Re}) \in \Gamma_{I}$ der vom kanonischen Morphismus $\psi: A \rightarrow \hat{A}$ induzierte Morphismus $\psi_{(\mathfrak{p}, \mathfrak{\beta})}: A_{\mathfrak{p}} \rightarrow \hat{A}_{\mathfrak{\beta}}$ formell glatt. $\left(D . h\right.$. ist $(\mathfrak{m}, \mathfrak{M}) \in \Gamma_{0}$ mit maximalem Ideal $\mathfrak{M}$, so lokalisiert die formelle Glattheit von $\psi_{(\mathrm{m}, \mathfrak{m})}$ in einer Teilmenge von $\left.\Gamma_{0}\right)$.

Beweis. Sei $(\mathfrak{p}, \mathfrak{P}) \in \Gamma_{I}$. Wähle $(\mathfrak{M}, \mathfrak{M}) \in \Gamma_{0}$ mit $\mathfrak{P} \subseteq \mathfrak{M}$ und $\mathfrak{M}$ maximal in $\operatorname{Spec}(\hat{A})$. Dann ist $\psi_{(\mathfrak{m}, \mathfrak{m})}: A_{\mathrm{m}} \rightarrow \hat{A}_{\mathfrak{m}}$ formell glatt, da $\hat{A}_{\mathfrak{R}} \simeq\left(A_{\mathrm{m}}\right)^{\wedge}$. Nach Voraussetzung ist $A / I$ quasiausgezeichnet; dann ist der von $\psi_{(\mathfrak{p}, \mathfrak{\beta})}$ induzierte Morphismus $\left.(A / I)_{\mathfrak{p}} \rightarrow \widehat{(A / I}\right)_{\mathfrak{p}}$ formell glatt, denn die formelle Glattheit von $(A / I)_{\mathfrak{m}} \rightarrow \widehat{(A / I)_{\mathfrak{M}}}$ lokalisiert. Da $\psi_{(\mathfrak{p}, \mathfrak{\beta})}$ flach ist, folgt dann mit [3] EGA $O_{I V}(19.7 .1)$ auch die formelle Glattheit von $\psi_{(\mathfrak{p}, \mathfrak{B})}$.

Folgerung 4.1. A/I sei quasiausgezeichnet. Dann ist für alle (p, $\mathfrak{P})$ $\in \Gamma_{I}$ der von $\psi_{(\mathfrak{p}, \mathfrak{\beta})}$ induzierte Morphismus der Komplettierungen $\varphi_{(\mathfrak{p}, \mathfrak{\beta})}: \hat{A}_{\mathrm{p}}$ $\rightarrow\left(\hat{A}_{\mathfrak{B}}\right)^{\wedge}$ formell glatt.

Beweis. Nach Lemma 4 ist $\psi_{(\mathfrak{p}, \mathfrak{\beta})}$ formell glatt. Die Behauptung folgt mit [3] EGA $O_{I V}$ (19.3.6).

Folgerung 4.2. A/I sei quasiausgezeichnet. Für alle $(\mathfrak{p}, \mathfrak{\Re}) \in \Gamma_{I}$ lokalisiert die formelle Glattheit von $\varphi_{(\mathfrak{p}, \mathfrak{\beta})}$. Insbesondere gilt für alle $(\mathfrak{p}, \mathfrak{\beta})$ $\in \Gamma_{1}$, wenn Spec $\varphi_{(\mathfrak{p}, \mathfrak{\beta})}$ die von $\varphi_{(\mathfrak{p}, \mathfrak{\beta})}$ induzierte Abbildung der Spektren: 
$\operatorname{Spec} \varphi_{(\mathfrak{p}, \mathfrak{\beta})}: \operatorname{Spec}\left(\left(\hat{A}_{\mathfrak{\beta}}\right)^{\wedge}\right) \rightarrow \operatorname{Spec}\left(\widehat{A_{\mathfrak{p}}}\right)$ bezeichnet

$$
\left(\operatorname{Spec} \varphi_{(\mathfrak{p}, \mathfrak{B})}\right)^{-1}\left(\operatorname{Reg}\left(\widehat{A_{\mathfrak{p}}}\right)\right)=\operatorname{Reg}\left(\left(\hat{A}_{\mathfrak{\beta}}\right)^{\wedge}\right) .
$$

Beweis. Die erste Behauptung folgt mit Theorem 2. Die zweite Behauptung ergibt sich aus der Regularität von $\varphi_{(\mathfrak{p}, \mathfrak{\beta})}$ (vgl. [3] EGA $O_{I V}$ (19.6.4) und (22.5.8)).

\section{§2. Das Hauptergebnis}

Theorem 3. A sei ein semilokaler noetherscher Ring, $I \subseteq \operatorname{rad}(A)$ ein im Jacobsonradikal von $A$ enthaltenes Ideal. A sei I-adisch komplett und A/I sei quasiausgezeichnet. Dann ist $A$ ebenfalls quasiausgezeichnet.

Beweis. Um (b) in Lemma $1 \mathrm{zu}$ zeigen, dürfen wir annehmen, daß $A$ ein Integritätsbereich ist. Wir haben dann nachzuweisen, daß für alle E $\in \operatorname{Sing}(\hat{A})$ \& $\cap A \neq(0)$ ist. Sei also $\& \in \operatorname{Sing}(\hat{A})$.

1. Fall. $\sqrt{\mathfrak{s}+I \hat{A}}=\bigcap_{i=1}^{s} \mathfrak{M}_{j}$, wobei die $\mathfrak{M}_{j}$ maximale Ideale in $\hat{A}$ sind. Dann folgt die Behauptung mit Lemma 3.

2. Fall. Es gibt ein nicht-maximales Primideal $\mathfrak{B} \in \operatorname{Spec}(\hat{A})$ mit $\mathfrak{P} \supseteq \mathbb{E}+I \hat{A}$.

In diesem Fall konstruieren wir ein geeignetes Ideal $\mathscr{D} \subseteq \mathscr{C}$ und zeigen: $\mathfrak{D} \cap A \neq(0)$.

Konstruktion von $\mathfrak{D}$. Für alle Paare $(\mathfrak{p}, \mathfrak{P}) \in \Gamma_{I}$ betrachten wir folgendes kommutative Diagramm kanonischer Morphismen:

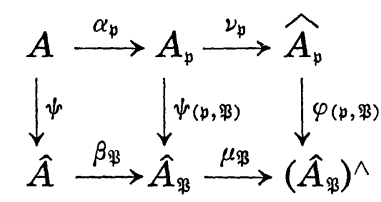

Für alle nichtmaximalen Primideale $\mathfrak{p} \in \operatorname{Spec}(A)$ mit $\mathfrak{p} \supseteq I$ definieren wir:

$$
\mathfrak{D}_{\mathfrak{p}}= \begin{cases}\bigcap_{\mathfrak{Q} \in \operatorname{Sing}\left(\hat{A_{\mathfrak{p}}}\right)} \mathfrak{Q} & \text { falls } \widehat{A_{\mathfrak{p}}} \text { nicht regulär } \\ \widehat{A_{\mathfrak{p}}} & \text { falls } \widehat{A_{\mathfrak{p}}} \text { regulär }\end{cases}
$$

und setzen: 


$$
\begin{aligned}
\mathfrak{D} & =\bigcap_{(\mathfrak{p}, \mathfrak{\beta}) \in \Gamma_{I}}\left(\mu_{\mathfrak{\beta}} \circ \beta_{\mathfrak{k}}\right)^{-1}\left(\varphi_{(\mathfrak{p}, \mathfrak{\beta})}\left(\mathfrak{D}_{\mathfrak{p}}\right)\left(\hat{A}_{\mathfrak{B}}\right)^{\wedge}\right) \\
& =\bigcap_{(\mathfrak{p}, \mathfrak{\beta}) \in \Gamma_{I}}\left[\mathfrak{D}_{\mathfrak{p}}\left(\hat{A}_{\mathfrak{P}}\right)^{\wedge} \cap \hat{A}\right]
\end{aligned}
$$

Behauptung 1. D ist ein reduziertes von Null verschiedenes Ideal in $\hat{A}$.

Beweis von Behauptung 1. Da nach Theorem 1 die formellen Fasern von $A$ geometrisch reduziert sind, ist $\widehat{A_{\mathfrak{p}}}$ reduziert für alle $\mathfrak{p} \in \operatorname{Spec}(A)$, denn nach Voraussetzung ist $A$ ein Integritätsbereich. Also ist $\mathfrak{D}_{p}$. entweder gleich $\widehat{A_{p}}$ oder ein reduziertes Ideal der Höhe $\geqq 1$. $\mathfrak{D}_{p}\left(\hat{A}_{\mathfrak{P}}\right)^{\wedge}$ ist dann für alle $(\mathfrak{p}, \mathfrak{P}) \in \Gamma_{I}$ ebenfalls reduziert (oder gleich $\left.\left(\hat{A}_{\mathfrak{B}}\right)^{\wedge}\right)$. Das ergibt sich wie folgt aus der Regularität von $\varphi_{(\mathfrak{p}, \mathfrak{B})}$ : Sei etwa $\mathfrak{D}_{\mathfrak{p}}=\bigcap_{i=1}^{n} \mathfrak{Q}_{i}$, $\mathfrak{Q}_{i} \in \operatorname{Spec}\left(\widehat{A_{\mathfrak{p}}}\right)$. Dann ist $\mathfrak{D}_{\mathfrak{p}}\left(\hat{A}_{\mathfrak{p}}\right)^{\wedge}=\bigcap_{i=1}^{n}\left[\mathfrak{Q}_{i}\left(\widehat{A}_{\mathfrak{B}}\right)^{\wedge}\right]$, und zu zeigen ist, daß für alle $(\mathfrak{p}, \mathfrak{\Re}) \in \Gamma_{I}$ und alle $\mathfrak{q} \in \operatorname{Spec}\left(\widehat{A_{\mathfrak{p}}}\right) \mathfrak{q}\left(\hat{A}_{\mathfrak{R}}\right)^{\wedge}$ reduziert ist. Mit [3] EGA $O_{I V}$ (19.7.1) folgt, daß der von $\varphi_{(\mathfrak{p}, \mathfrak{\beta})}$ induzierte Morphismus der Restklassenringe

$$
\widehat{A_{p}} / \mathfrak{q} \rightarrow\left(\widehat{A_{p}}\right)^{\wedge} / \mathfrak{q}\left(\hat{A_{p}}\right)^{\wedge}
$$

ebenfalls regulär ist. Daraus ergibt sich unmittelbar, daß $\left(\hat{A}_{\mathfrak{p}}\right)^{\wedge} / \mathfrak{q}\left(\hat{A}_{\mathfrak{p}}\right)^{\wedge}$ die Serreschen Kriterien $\left(R_{0}\right)$ und $\left(S_{1}\right)$ erfüllt. Also ist $\mathscr{D}_{p}\left(\hat{A}_{\mathfrak{p}}\right)^{\wedge}$ reduziert und -nach Konstruktion von $\mathfrak{D}_{\mathfrak{p}}$-Durchschnitt von Primidealen aus dem singulären Ort von $\left(\hat{A}_{\mathfrak{\beta}}\right)^{\wedge}\left(\right.$ denn $\varphi_{(\mathfrak{p}, \mathfrak{\beta})}$ ist insbesondere treuflach). Damit. folgt, daß $\cong$ Durchschnitt von Primidealen aus dem singulären Ort von $\hat{A}$ ist. Da $\hat{A}$ nach Theorem 1 reduziert ist, folgt Behauptung 1 .

Behauptung 2.

$$
\mathfrak{D}_{\mathfrak{p}}\left(\hat{A}_{\mathfrak{P}}\right)^{\wedge}= \begin{cases}\left(\hat{A}_{\mathfrak{P}}\right)^{\wedge} & \text { falls }\left(\hat{A}_{\mathfrak{R}}\right)^{\wedge} \text { regulär } \\ \bigcap_{\mathfrak{Q} \in \operatorname{Sing}\left(\hat{A}_{\mathfrak{P}}\right) \wedge} \cong & \text { falls }\left(\hat{A}_{\mathfrak{P}}\right)^{\wedge} \text { singulär }\end{cases}
$$

Beweis von Behauptung 2. Wie im Beweis von Behauptung 1 gezeigt,

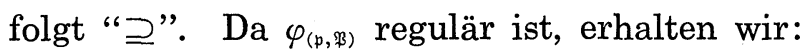

$$
\varphi_{(\mathfrak{p}, \mathfrak{R})}^{-1}\left(\bigcap_{\varrho \in \operatorname{Sing}\left(\left(\hat{A}_{\mathfrak{P}}\right) \wedge\right.} \mathfrak{Q}\right) \supseteq \mathfrak{D}_{\mathfrak{p}}=\bigcap_{\mathfrak{q} \in \operatorname{Sing}\left(\hat{A_{\mathfrak{p}}}\right)} \mathfrak{q}
$$

und es folgt Behauptung 2.

Behauptung 3. 도․

Beweis von Behauptung 3. $\mathfrak{B} \in \operatorname{Spec}(\hat{A})$ sei ein nichtmaximales Primi- 
deal, das $\mathfrak{S}+I \hat{A}$ umfaßt. Mit $\mathfrak{p}=A \cap \mathfrak{P}$ ist dann $(\mathfrak{p}, \mathfrak{P}) \in \Gamma_{I}$, und wegen $\mathfrak{F} \in \operatorname{Sing}(\hat{A})$ ist $\mathbb{E}\left(\hat{A}_{\mathfrak{\beta}}\right)^{\wedge}$ Durchschnitt von Primidealen aus dem singulären Ort von $\left(\hat{A}_{\mathfrak{B}}\right)^{\wedge}$. Mit Behauptung 2 ergibt sich nun:

$$
\mathfrak{P}_{\mathfrak{p}}\left(\hat{A}_{\mathfrak{B}}\right)^{\wedge} \subseteq \mathfrak{E}\left(\hat{A}_{\mathfrak{B}}\right)^{\wedge}
$$

und es folgt Behauptung 3 .

Behauptung 4. $\mathfrak{Q}$ sei ein $\mathfrak{Q}+I^{n} \hat{A}$ umfassendes Primärideal in $\hat{A}$. Dann folgt: ( $\cap A) \hat{A} \supseteq \mathfrak{D}+I^{n} \hat{A}$ (dabei ist $n \in N$ beliebig, aber fest).

Beweis von Behauptung 4. $\mathfrak{O}$ sei $\mathfrak{\beta}$-primär mit $\mathfrak{\beta} \in \operatorname{Spec}(\hat{A})$. Ist $\mathfrak{P}$ maximal in $\operatorname{Spec}(\hat{A})$, so ist nichts zu zeigen. Sei also $\mathfrak{\beta}$ nicht maximal; dann ist mit $\mathfrak{p}=\mathfrak{B} \cap A(\mathfrak{p}, \mathfrak{B}) \in \Gamma_{I}$. Wir setzen $\mathfrak{q}=\mathfrak{Q} \cap A$ und betrachten wieder folgendes kommutative Diagramm:

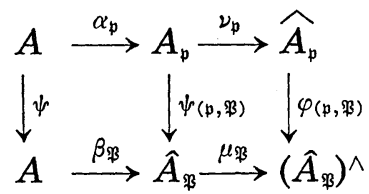

$\mathrm{Da} \mathfrak{O} \mathfrak{\Re - p r i m a ̈ r}$ ist, erhalten wir:

$$
\mathfrak{q}=\mathfrak{Q} \cap A=\mathfrak{Q}\left(\hat{A}_{\mathfrak{\beta}}\right) \wedge \cap A .
$$

(4.1) $\mathfrak{Q}\left(\hat{A_{\mathfrak{p}}}\right)^{\wedge} \cap \widehat{A_{\mathfrak{p}}}$ ist ein $\mathfrak{p} \widehat{A_{\mathfrak{p}}}$-primäres Ideal, das $\mathfrak{D}_{\mathfrak{p}}+I^{n} \widehat{A_{\mathfrak{p}}}$ umfaßt .

Beweis von (4.1). $\mathscr{D}\left(\hat{A}_{\mathfrak{\beta}}\right)^{\wedge}$ ist ein Durchschnitt von Primidealen aus dem singulären Ort von $\left(\hat{A}_{\mathfrak{\beta}}\right)^{\wedge}$. Nach Behauptung 2 gilt dann: $\mathscr{D}\left(\hat{A}_{\mathfrak{\beta}}\right)^{\wedge}$ $\supseteq \mathfrak{D}_{\mathfrak{p}}\left(\hat{A}_{\mathfrak{\beta}}\right)^{\wedge}$. Da $\mathfrak{S}+I^{n} \hat{A}$ in $\mathfrak{Q}$ enthalten ist, erhalten wir damit $\mathfrak{Q}\left(\hat{A}_{\mathfrak{\beta}}\right)^{\wedge}$ $\cap \widehat{A_{\mathfrak{p}}} \supseteq \mathfrak{D}_{\mathfrak{p}}+I^{n} \widehat{A_{\mathfrak{p}}} . \quad$ Ferner ist $\mathfrak{Q}\left(\hat{A}_{\mathfrak{P}}\right)^{\wedge} \mathfrak{P}\left(\hat{A}_{\mathfrak{B}}\right)^{\wedge}$-primär, also ist auch $\mathfrak{Q}\left(\hat{A}_{\mathfrak{P}}\right)^{\wedge}$ $\cap \widehat{\hat{A}_{\mathfrak{p}}} \mathfrak{p} \widehat{A_{p}}$-primär.

$$
\mathfrak{q} \widehat{A_{\mathfrak{p}}}=\mathfrak{\Re ( \widehat { A _ { \mathfrak { p } } } )} \wedge \cap \widehat{A_{\mathfrak{p}}}
$$

Der Beweis von (4.2) ergibt sich unmittelbar aus der Tatsache, daß $\mathfrak{Q}\left(\hat{A}_{\mathfrak{p}}\right) \wedge \cap \widehat{A_{\mathfrak{p}}} \mathfrak{p} \widehat{A_{\mathfrak{p}}}$-primär ist.

$$
\mathfrak{q} \hat{A} \supseteq \mathfrak{D}+I^{n} \hat{A} .
$$

Beweis von (4.3). $\mathrm{Da} \mathfrak{q} \mathfrak{p}$-primär ist, ist $\operatorname{Ass}(\hat{A} / \mathfrak{q} \hat{A})=\left\{\mathfrak{P}_{1}, \cdots, \mathfrak{\beta}_{m}\right\}$, wobei die $\mathfrak{P}_{i}$ die minimalen Primoberideale von $\mathfrak{p} \hat{A}$ in $\operatorname{Spec}(\hat{A})$ sind. Das ergibt sich sofort mit Theorem 1 aus [2] Chap. IV, $\S 2$, no. 6, Theorem 
2. Also ist $\mathfrak{q} \hat{A}=\mathfrak{r}_{1} \cap \cdots \cap \mathfrak{r}_{m}$, wobei die $\mathfrak{r}_{i} \mathfrak{\beta}_{i}$-primär sind. Wegen $\left(\mathfrak{p}, \mathfrak{P}_{i}\right) \in \Gamma_{I}$ betrachten wir wieder folgendes kommutative Diagramm:

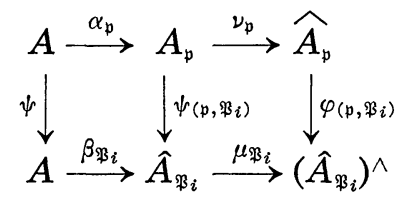

Mit (4.1) und (4.2) folgt: $\mathfrak{q}\left(\hat{A}_{\mathfrak{P}_{i}}\right)^{\wedge}=\mathfrak{x}_{i}\left(\hat{A}_{\mathfrak{P}_{i}}\right)^{\wedge} \supseteq \mathfrak{D}_{\mathfrak{p}}\left(\hat{A}_{\mathfrak{P}_{i}}\right)^{\wedge}$. Daraus ergibt sich, daß $\mathfrak{D}+I^{n} \hat{A}$ in $\mathfrak{r}_{i}=\mathfrak{q}\left(\hat{A}_{\mathfrak{B}_{i}}\right)^{\wedge} \cap \hat{A}$ enthalten ist. Insgesamt folgt die Behauptung 4: $\mathfrak{A} \supseteq \supseteq \mathfrak{D}+I^{n} \hat{A}$. Da die Behauptung 4 insbesondere für die Primärkomponenten von $\mathfrak{D}+I^{n} \hat{A}$ erfüllt ist, folgt fur alle $n \in N$ : $\left[\left(\mathfrak{D}+I^{n} \hat{A}\right) \cap A\right] \hat{A}=\mathfrak{D}+I^{n} \hat{A}$, und die Behauptung des Satzes ergibt sich aus Lemma 2.

\section{Folgerungen.}

(3.1) A sei ein semilokaler quasiausgezeichneter Ring. Dann ist der formale Potenzreihenring $A\left[\left[T_{1}, \cdots, T_{n}\right]\right]$ in endlich vielen Unbestimmten ebenfalls quasiausgezeichnet.

(3.2) A sei ein semilokaler quasiausgezeichneter Ring, $I \subseteq \operatorname{rad}(A)$ ein im Jacobsonradikal von A enthaltenes Ideal. Dann ist die I-adische Komplettierung von A wieder quasiausgezeichnet.

\section{LITERATUR}

[1] André, M., Localisation de la lissite formelle, Manuscripta Math. 13 (1974), 297307.

[2] Bourbaki, N., Elements of Mathematics: Commutative Algebra, Paris, Hermann, (1972).

[ 3 ] Grothendieck, A., Éléments de Géométrie algébrique, Inst. haut. Étud. sci., Publ. math. 20 (1964).

[4] —_ Éléments de Géométrie algébrique, Inst. haut. Étud. sci., Publ. math. 24 (1965).

[5] Matsumura, H., Commutative Algebra, New York, Benjamin (1970).

[6] — Formal power series rings over polynomial rings I, in: Number Theory, Algebraic Geometry and Commutative Algebra, in honour of Y. Akizuki, Tokyo, Kinokuniya (1973), 511-520.

[ 7 ] Marot, J., Sur les anneaux universellement japonais, Bull. Soc. math. France 103 (1975) , 103-111.

[ 8 ] Nomura, M., Formal power series rings over polynomial rings II, in: Number Theory, Algebraic Geometry and Commutative Algebra, in honour of Y. Akizuki, Tokyo, Kinokuniya (1973), 521-528.

[ 9 ] Rotthaus, C., Nicht ausgezeichnete, universell japanische Ringe, Math. Z. 152 (1977), 107-125. 
[10] Rotthaus, C., Universell japanische Ringe mit nicht offenem regulärem Ort, Nagoya Math. J. 74 (1979), 123-135.

[11] Valabrega, P., A few theorems on completion of excellent rings, Nagoya Math. J. 61 (1976), 127-133.

Mathematisches Institut der Universität Münster 